\title{
El desarrollo del lenguaje en un caso de carencias afectivas graves en la primera infancia
}

\author{
GRACE SHUM Y ANGELES CONDE \\ Universidad Complutense de Madrid
}

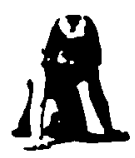

\section{Resumen}

Los datos lingü̈́sticos fueron registrados mediante un video en un contexto natural y babitual del niño. Se analiza el lenguaje desde los tres planos lingüisticos, sintáctico, semántico y pragmático. Además, para observar el nivel de desarrollo lingüistico, se comparó la producción del lenguaje del sujeto estudiado con la de otro niño cuyo desarrollo es adecuado.

Tras los resultados obtenidos del trabajo, se puede concluir que los factores externos, en los aspectos que están relacionados a pantas de cuidado y de interacción diádica adulto-niño, son fundamentales para la adecuada adquisición y desarrollo lingiiistico y, además, la rebabilitación del mismo no es muy esperanzadora.

Palabras clave: Adquisición del lenguaje; Maltrato del niño.

\section{Language Development in a Case of Serious Affective Disturbance in Early Childhood}

\section{Abstract}

The present study deals with the acquisition and development of language in a small child (from 2;6 to 4;6 years old) who bad been abused from birth up to the age of two. The child's language was recorded in a natural context at monthly intervals, each session lasting 30 mins. Language production was analyzed from three levels: Syntactic, semantic, and pragmatic. It was then compared with the expressive language of a cbild with nomal development to assess the subject's language development. From the results, it is concluded that external factors, mainly tbose aspects related to caretaker-infant interaction patterns, are essential for the correct acquisition and development of language; furthermore, delays in this area are difficult to overcome.

Key words: Language acquisition; Child abuse.

Correspondencia con autor: Dpto. de Psicología Evolutiva y de la Educación. Universidad Complutense de Madrid. Campus de Somosaguas. 28023 Madrid 


\section{INTRODUCCION}

El desarrollo infantil además de las condiciones referidas a la maduración psicológica y orgánica, necesita también de fenómenos externos como el medio ambiente físico y social. Dentro de los factores externos, los aspectos relacionados con las pautas de cuidados, de socialización del niño con su entorno (la comunicación diádica adulto-niño) son fundamentales, puesto que, por la naturaleza social que el niño posee, su desarrollo tiene que situarse, necesariamente, en los procesos comunicativos. En la interacción adulto-niño, el adulto es el que desempeña un papel fundamental, asumiendo no sólo las tareas de satisfacer las necesidades fisiológicas del pequeño, sino haciendo de organizador y transmisor de la pautas culturales, instruyendo y proporcionando al pequeño las experiencias necesarias. Por consiguiente, si las pautas y contextos interactivos no son adecuados, aparecerán problemas en el desarrollo infantil, Tizard, 1972; Loutre, 1981; Bowlby, 1969, 1976; Alinsworth, 1969; López; 1977; etcétera.

No obstante, el niño no es un ser pasivo en el proceso comunicativo. Autores como Bruner, 1986; Shaffer, 1977; Schaffer y Crook, 1981; Turner, 1980; etc., afirman que la interacción adulto-niño es un proceso dialéctico niño-medio. Esto supone que, a través de la interacción niño-adulto, el agente socializador contribuye al desarrollo infantil y, a su vez, el niño modifica y transforma el contexto comunicativo - en función de su desarrollo evolutivo- desempeñando, progresivamente, un papel más activo en la comunicación con el adulto. Así pues, puede decirse que el adecuado desarrollo infantil necesita de unas determinadas pautas interactivas adulto-niño. Según Kaye (1986) son esenciales para el desarrollo infantil las siguientes condiciones:

- La actitud de los padres ante el bebé. Es decir, entender a éste como parte activa (aunque en realidad no lo sea) del sistema familiar, haciéndole intervenir en diálogos, juegos y otras interacciones significativas que le permitan integrarse, poco a poco, en el mundo adulto.

- Cierta regularidad en los ciclos internos del bebé, respecto a la alimentación, sueño, juegos, etcétera.

- Interacciones sociales que estimulen al bebé (alcanzar objetos, situaciones cara a cara, emitir sonidos y palabras, etc.).

En resumen, el papel del adulto es fundamental en el proceso de socialización infantil, ya que organiza la experiencia del niño, haciendo posible y guiando el desarrollo global, y más específicamente el del lenguaje. Como señala Bruner (1984) la relación habitual adulto-niño constituye la diada que permite al niño aprender todo aquello relacionado con la comunicación. De esta manera, el niño recibe las claves del lenguaje al participar de un tipo de relación social que actúa de modo consonante con el discurso.

Diversas circunstancias ambientales han sido descritas como potencialmente patógenas para el normal desarrollo del niño, tales como la separación de la madre, la existencia de varias figuras maternas, las enfermedades psiquiátricas de los padres, la institucionalización y, en último término, el maltrato del niño en su doble vertiente de agresión física y de abandono, falta de afecto y descuido. Estas circunstancias ambientales anómalas van a tener una repercusión tanto más adversa sobre la conducta del niño cuanto más pequeño sea éste y cuanto más prolongada sea su duración.

Todos estos déficits se manifiestan de diferentes modos en el comportamiento infantil. Así, las funciones cognitivas y el lenguaje son los más vulnerables y los que 
tienen un peor pronóstico, mientras que la motricidad presenta una mejor evolución (Tizard, 1972;, Pawell, 1980; Mardomingo, 1981; López, 1981; Robertson y Milner, 1987). El desarrollo físico (peso, talla, maduración ósea, etc.) también se encuentra afectado, como se demuestra en numerosos estudios realizados a partir de la década de los sesenta (Ainsworth, 1962; Gardner, 1976).

Así pues, los problemas en el desarrollo de niños maltratados y/o abandonados son muy complejos. No obstante, en este estudio se plantea un análisis más profundo en el aspecto de adquisición y desarrollo del lenguaje de un niño que recibió malos tratos en sus primeros dos años de vida, posteriormente, fue ingresado en una institución de protección de menores y, finalmente, fue adoptado.

El motivo de elegir el estudio de este aspecto del desarrollo del niño se debe, por un lado, a la escasez de trabajos de investigación sobre este ámbito específico y, por otro, porque el lenguaje no sólo es vehículo de comunicación, sino también base de desarrollo de las funciones psicológicas superiores (Vigotski, 1973; Luria y Yudovich, 1978). Por consiguiente, los problemas que aparecen en el desarrollo lingüístico del niño reflejan, a su vez, problemas en otras áreas.

\section{METODO}

Proponemos un estudio empírico, cuyo objetivo es identificar las pautas de desarrollo del lenguaje infantil en un niño que ha recibido malos tratos y deprivación afectiva en sus primeros años de vida y que posteriormente fue adoptado.

\section{SUJETOS}

El presente trabajo consiste en un estudio longitudinal con un niño-Miguel-, en un período de tiempo que va desde los $2 ; 6$ años (etapa en la que se encuentra viviendo en una institución de protección de menores), hasta los 4;6 años (nueve meses después de haber sido adoptado por una familia).

Este niño había sido maltratado, tanto física como psíquicamente (falta de afecto, abandono, falta de cuidado, etc.), desde su nacimiento hasta el momento en que fue institucionalizado (2;6 años). Después de permanecer un año en una institución, fue adoptado a los 3;6 años.

Para poder llevar a cabo comparaciones del desarrollo del lenguaje del niño estudiado en sus diversos niveles (sintáctico, semántico y pragmático) utilizamos datos lingüísticos de otro niño (Carlos), cuyo desarrollo es considerado adecuado como ya se ha mostrado en trabajos anteriores (Shum, 1986; Shum y, otros 1988a, 1988b).

\section{PROCEDIMIENTO}

Aunque el objetivo principal de este trabajo es el estudio del lenguaje, como ya se especificó anteriormente, nos pareció importante realizar algunas evaluaciones en aspectos físicos y psicológicos, para mostrar el efecto de las carencias afectivas en la primera infancia. Para ello, se llevó a cabo un examen neurológico en el cual no se detectó ninguna lesión cerebral y una serie de pruebas de carácter físico y psicológico en dos etapas diferentes.

- en el comienzo del régimen de institucionalización, 
- encontrándose en régimen de adopción (nueve meses después).

Se utilizaron las gráficas de peso y talla de Children's Hospital of Boston, adaptadas a la población española; la determinación de la edad ósea se ha realizado a través de las tablas de Greulich y Pyle, tras hacer radiografía de la muñeca izquierda. Asimismo se realizaron controles de las cifras de calcio, fósforo y fosfatasa alcalina en sangre, así como radiografía de cráneo y medición de perímetro cefálico. Todos estos controles se han llevado a cabo en el comienzo del régimen de institucionalización, y a los nueve meses de adopción.

En cuanto al desarrollo psicológico del niño, se ha estudiado a través de la aplicación de las Escalas McCarthy de Aptitudes y Psicomotricidad para niños, también en dos ocasiones, en el período institucional ( $3 ; 3$ años) y posteriormente en el periodo de adopción (4;2 años).

En la primera etapa de la vida institucional de Miguel, dada la imposibilidad de recoger información sobre la producción verbal del niño, ya que ésta no existía, se realizaron grabaciones semanales a través de vídeo, durante un período de tres meses (desde los 34 a los 36 meses de edad). Estas observaciones asistemáticas tuvieron lugar en la guardería del centro donde vivía el niño. Dada la imposibilidad de categorizar la producción linguística del sujeto, se realizó un análisis descriptivo de sus pautas de comportamiento comunicativo y social que tuvieron lugar en los juegos e interacciones con sus compañeros y educadores.

Con respecto al estudio del lenguaje verbal, objerivo fundamental del presente estudio, se recogieron muestras del lenguaje de Miguel a través de observación natural. Se registraron, con la ayuda de una cámara de vídeo, sesiones de interacción adulto-niño de una duración de 30 minutos y con una periodicidad mensual. El niño, durante estas sesiones, jugaba con un adulto. Este adulto era una de las observadoras con la que previamente se había familiarizado (durante el período transcurrido en la institución) y su madre adoptiva (en el período que denominamos posterior a la adopción). El hecho de que no se hayan utilizado para el análisis del lenguaje, registros que se han tomado con sus cuidadores, se debe a que dichos registros no son adecuados para el estudio de la producción lingüística del niño, ya que las muestras de la interacción y del lenguaje del sujeto eran más pobres que las registradas con el observador. Esto se debe fundamentalmente a que la interacción diádica adulto-niño en la vida institucional es prácticamente inexistente por causa de la propia estructura de la institución (el número de niños, el continuo cambio de cuidadores, etc.). Mientras que la interacción que tiene lugar con la observadora posibilita una comunicación diádica propiamente dicha, debido a que esta misma experimentadora realizaba con el niño sesiones de habilitación lingüística y comunicativa varias veces por semana. Las situaciones interactivas no eran estructuradas, eran juegos espontáneos con juguetes conocidos para Miguel, tales como coches, construcción, cuentos, etc.

Posteriormente, se transcribieron íntegramente los enunciados emitidos, tanto por el niño como por el adulto, así como las situaciones del contexto comunicativo necesarias para comprender los mismos. A partir de estas transcripciones se analizó la producción verbal del niño en tres aspectos lingüísticos: sintáctico, semántico y pragmático.

Como indicador del desarrollo global del lenguaje se utilizó la Longitud Media de los Enunciados (LME). Aunque esta medida presenta ciertas limitaciones, nos sirvió como índice aproximado de la evolución y desarrollo del lenguaje del niño. Para calcular este índice, hemos aplicado un código adaptado a los morfemas de la Lengua Castellana (Vila, 1985; Shum, 1986).

En el plano morfosintáctico se analizó a través de las categorías y subcategorías 
gramaticales del español: artículo, sustantivo, verbo, adjetivo, adverbio, preposición y conjunción. Asimismo se clasificaron como subcategorías las referidas a algunos accidentes gramaticales: género, número y tiempo. Para todas ellas se tuvo en cuenta el momento de aparición en la producción lingüística del niño, así como la forma de uso, estableciendo, además, comparación con el desarrollo lingüístico de un niño cuyo desarrollo del lenguaje es considerado adecuado. Además, fueron analizados aquellos mensajes verbales en los que aparecían errores morfosintácticos, de acuerdo a las siguientes categorías:

- Concordancia: Se trata de los enunciados en los que aparecen problemas referidos a las relaciones de género, número y persona, entre los elementos de la oración.

- Omisión: Se refiere a la ausencia de elementos morfológicos que contravienen las reglas gramaticales.

- Sustitución: Cambio de un elemento verbal por otro dentro de la misma categoría gramatical, o bien cambio de elementos pertenecientes a distintas funciones gramaticales.

- Adición: Introducción en un enunciado de cualquier categoría morfológica que no aporte ninguna información nueva, infringiendo, además, reglas gramaticales.

El aspecto semántico fue analizado a través de la evolución lexical, haciendo un análisis de las palabras diferentes producidas por el niño en cada observación. Esta forma de medida de la evolución lexical es muy difícil de controlar durante un periodo de tiempo tan extenso, en niños con un desarrollo del lenguaje adecuado. Sin embargo, dadas las características de la adquisición del lenguaje de este sujeto, nos puede resultar indicativa. En este aspecto, fueron también analizados errores en la expresión verbal de Miguel. Estos errores son del tipo de inadecuación del tiempo verbal en relación con el contexto y con la propia expresión del niño, errores del tipo de conocimiento físico y social. Nos parece interesante este análisis, ya que es un buen indicador de conocimiento semántico, riqueza lexical y, de algún modo, de conocimiento general del niño.

El desarrollo pragmático se evaluó mediante un sistema de categorías referidas a las funciones que desempeñan los enunciados del niño en las secuencias de interacción con el adulto. Así, se dividieron en: enunciativa, de demanda y fática.

Por último, se analizaron los mensajes no adecuados pragmáticamente. Estos se refieren a los mensajes del niño que no responden de forma adecuada a los del adulto en la secuencia interactiva correspondiente, aunque pueden ser correctos sintáctica y semánticamente. Esta inadecuación funcional de las expresiones infantiles puede deberse a un problema de atención al mensaje emitido por su interlocutor, o bien a problemas de comprensión del lenguaje del adulto por parte del niño, o de la situación contextual donde expresa el mensaje. Si eliminamos los mensajes infantiles que evidencian un problema de atención, podemos concluir que los enunciados inadecuados emitidos por el niño serán muestra del déficit lingüístico de éste, bien comprensivo o bien de adecuación de su expresión verbal al contexto (de carácter pragmático).

Además, para relacionar el desarrollo lingüístico con el desarrollo cognitivo, tuvimos en cuenta si el objeto y/o la acción a la que hacía referencia el niño tenía que ver con el contexto en el que se producía dicha expresión verbal. Así, se establecieron las siguientes categorías:

A. Enunciativa: 1. Referencia a objeto y/o acción presente (ROP)

2. Referencia a objeto ausente (ROA) y/o acción pasada o futura.

Estos mensajes son enunciados infantiles que denominan objetos o hacen refe- 
rencia a acciones distinguiendo si el objeto está presente en el contexto comunicativo o no. Asimismo, si se trata de acciones que están ocurriendo en el momento de la comunicación, o bien han ocurrido con anterioridad o se refieren a acciones hipotéticas o futuras.

B. Demanda: 1. Acción y/o objeto (DOP).

2. Información (DI): a, con interrogación abierta; b, con interrogación cerrada.

3. Ayuda (DA).

En esta categoría incluimos los mensajes del niño que solicitan algo del adulto, ya sea un objeto, una acción o una información.

C. Fática: son aquellos enunciados que cumplen sólo la función de establecer o mantener contacto con el interlocutor.

D. Inadecuación Pragmática: son aquellos enunciados que no responde a los mensajes del interlocutor en las secuencias interactivas, aunque la forma sintáctica y semántica sea correcta.

\section{RESULTADOS}

\section{Análisis de los aspectos fisicos y psicológicos del desarrollo del niño}

Los resultados obtenidos tras los análisis en los aspectos físicos y psicológicos son los siguientes:

- El perímetro cefálico se encontraba en el primer control (26 meses) a +2SD (98\%), habiéndose producido una disminución del mismo a los cuatro años.

- El peso ha pasado del percentil 25 al 50 en el segundo control (4;6 años). La altura, que se encontraba entre el percentil 10 y 25 , ha pasado al 25.

- Los datos analíticos que se observan en el primer control (hipocalcemia, hipofosfatemia y fosfatasa alcalina alta) correspondientes a raquitismo (síndrome propio de niños maltratados; Mardomingo, 1981) se han normalizado en el segundo análisis. La edad ósea, que presentaba un retraso de nueve meses cuando se realizaba el primer control, se corresponde con su edad cronológica en el segundo control.

En resumen se puede decir que el síndrome de raquitismo que presentaba Miguel en el período correspondiente a su ingreso en la institución, se encuentra plenamente superado en el segundo control, nueve meses después de que fuese adoptado.

En la primera aplicación de las Escalas McCarthy, Miguel presentaba un retraso golbal en el desarrollo, con una puntuación típica General Cognitiva (GC) de 74 y una puntuación verbal de 34. En el segundo control (4;2 años) la puntuación (GC) ha pasado a 72 , mientras que la verbal es de 38 . Por tanto, ha habido una mejoría en el lenguaje de 4 puntos. Se ha producido también una mejoría en la Escala de Memoria de 2 puntos, mientras que han descendido las puntuaciones perceptivomanipulativas y de motricidad. Puede concluirse que Miguel ha mejorado en edad mental pero no de manera paralela a su edad cronológica, lo cual explica que el desarrollo global del niño sea en conjunto numéricamente más bajo (Gráfico 1).

Con respecto a las habilidades comunicativas, en la primera etapa del estudio se observa en el niño una expresión gestual pobre, fácilmente inexpresivo y mirada triste, sin ningún punto de referencia específica. Raramente ofrecía contacto visual, tanto con personas como con objetos. No aparecía lenguaje verbal propiamente dicho, existían sonidos articulados en situaciones específicas. Debido a la rigidez de los movimientos y a su inexpresividad facial, su lenguaje analógico es muy pobre. A 
su vez, sus juegos son simples y repetitivos; frecuentemente establecía relación con uno o dos objetos. El desarrollo social de Miguel era también muy reducido. No mostraba ninguna necesidad de buscar contacto con otra persona, ni con un adulto ni tampoco con sus iguales para jugar. Inicialmente, no aparecía ninguna señal que indicase una intención clara de interacción, ni grupal ni interpersonal, excepto respuesta de temblor y de angustia ante personas y situaciones extrañas.

Figura 1

Escalas McCartby de aptitudes y psicomotricidad para niños

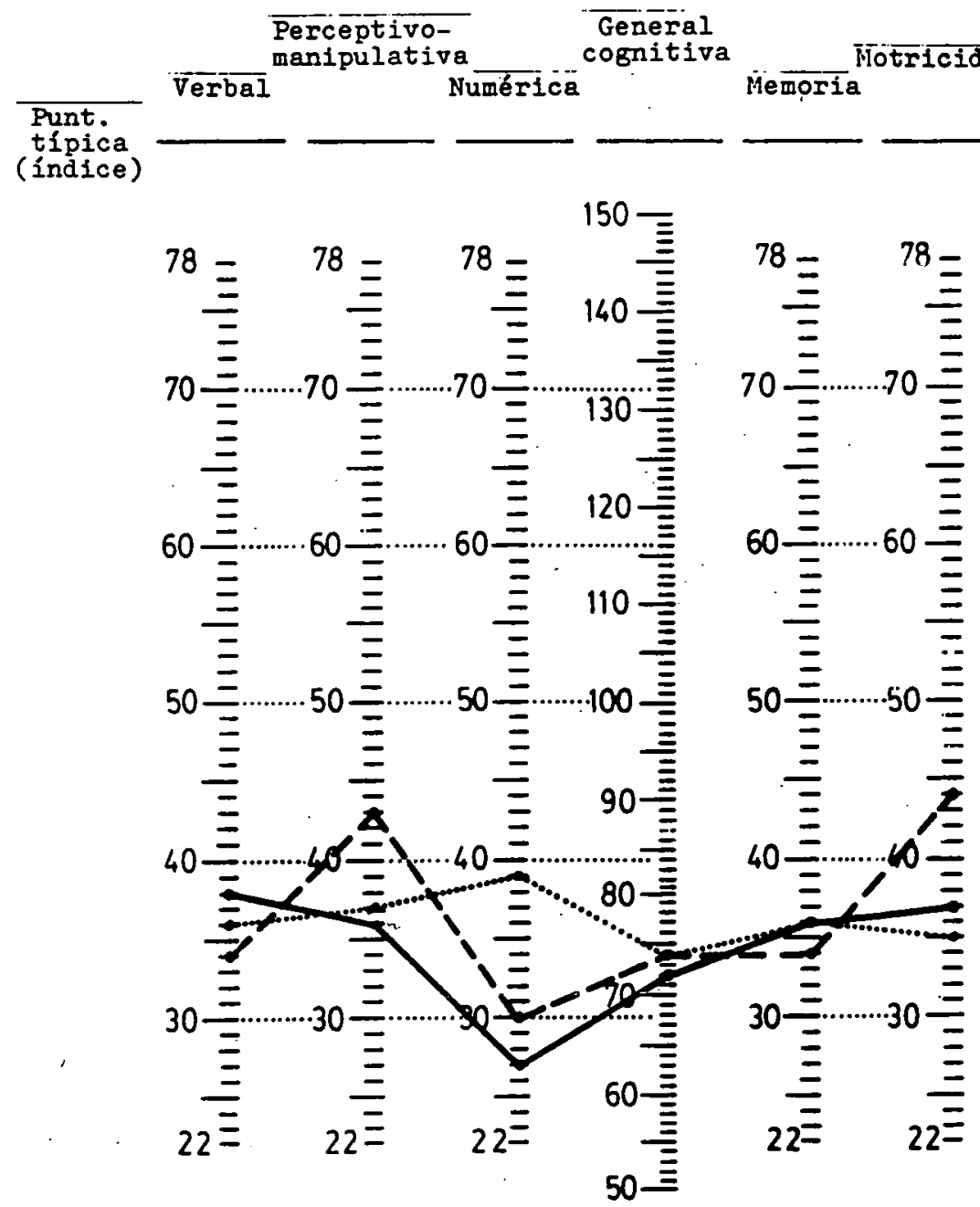

$-\ldots--3$ años 3 meses

4 años 2 meses

\section{6 años 3 meses}

Así, encontramos en esta etapa una deficiente capacidad comunicativa y graves déficits en el desarrollo del lenguaje verbal, ya que a los tres años de edad el niño aún no lo utilizaba. 


\section{Análisis global del lenguaje a través de la Longitud Media de los Enunciados}

Con respecto al desarrollo global del lenguaje, se observa que las puntuaciones de la Longitud Media de los Enunciados (LME) producidas por Miguel son mucho más bajas que las correspondientes a un niño de su edad. Como puede verse en la Tabla I la puntuación máxima obtenida por el niño estudiado (2,24 puntos a los 3;11 años) no alcanza a la obtenida por otro niño (Carlos), cuyo desarrollo del lenguaje es adecuado (como ya se ha mostrado en estudios anteriores; Shum, 1986, 1988) a los dos años de edad. La tendencia de la evolución de la LME en el caso de Carlos es ascendente, mientras que en Miguel prácticamente se mantiene estable, aun tratándose de una edad superior en la que tenía que darse una evidencia de desarrollo mayor. Esto significa que el lenguaje de Miguel en el período comprendido entre los tres y cuatro años de edad no ha superado todavía la etapa telegráfica, ya que presenta enunciados propios de niños de dos años y no modifica su estructura durante todo el tiempo de observación.

TABLA I

Evolución de la longitud media de los enunciados (LME)

\begin{tabular}{lccc}
\hline & MIGUEL & & \multicolumn{2}{c}{ CARLOS } \\
EDAD & LME & EDAD & LME \\
\hline & & $2 ; 0$ & 2,50 \\
& & $2 ; 3$ & 3,38 \\
& & $2 ; 6$ & 3,51 \\
$3 ; 0$ & 1,45 & $2 ; 9$ & 3,84 \\
$3 ; 2$ & 1,47 & $3 ; 0$ & 4,10 \\
$3 ; 11$ & 2,24 & & \\
$4 ; 4$ & 1,86 & & \\
\hline
\end{tabular}

\section{Análisis Morfosintáctico del Lenguaje}

Realizando un análisis cualitativo del lenguaje producido por Miguel, en líneas generales se puede decir que aparecen notables diferencias en relación con el lenguaje del niño con desarrollo adecuado, en cuanto a las características morfosintácticas se refiere.

Con respecto a la caregoría morfológica verbal, encontramos que no aparece el tiempo pasado hasta los 4;4 años; además, los verbos producidos por el niño suelen aparecer conjugados en primera persona del singular, excepto algunos verbos de uso muy frecuente, como «ser», «estar», «salir», etc., que también son utilizados en tercera persona del singular. La segunda persona del singular sólo es usada en imperativo, como se puede observar en los siguientes ejemplos:

- Que yo tengo un ten (tren) a casa/que era de vía ( $4 ; 4$ años).

- Mamá/mira (3;11 años).

En cuanto al uso de pronombres se puede decir que el niño sólo utiliza habitualmente los pronombres demostrativos; no aparecen, en general, los plurales y la aparición de los pronombres personales (cuyo uso es muy precoz en niños con desarrollo adecuado; Shum y otros, 1988b) es también muy tardía en el niño estudiado.

El adjetivo es utilizado por el niño sólo en su forma calificativa (referida a los 
tamaños «grande» o "pequeño») e indefinida ("otro", "todo»). Aunque, como se observa en los siguiente ejemplos, Miguel no conoce su significado correcto:

A. ¿Son grandes o pequeños?

N. Grandes/pequeños (3;11 años).

A. ¿Qué más cosas tienen los niños?

N. Otra tripa (4;4 años).

Se puede decir que el artículo es una de las categorías morfológicas que presenta menor problema en el lenguaje de Miguel, ya que aparecen en toda sus formas desde, prácticamente, el primer momento.

El adverbio comienza siendo utilizado como indicación de lugar o modo: «así», «aquí" aparecen a los 3;11 años; «dentro» a los 4;4 años. Los otros adverbios que aparecen de modo esporádico: «ahora», «luego», son generalmente imitaciones del lenguaje del adulto. Esto contrasta con el uso que hacen otros niños, en los que el uso de adverbio comienza a ser progresivo a partir de los dos años de edad (Shum, 1988). Mientras que en Miguel son usados de forma meramente deíctica y contextual, como se observa en los siguientes ejemplos:

A. ¿Y cómo se lleva?/¿cómo se lleva esta niña a la otra?

N. Así(3;11 años).

A. Hace mucho tiempo que nos conocemos/¿te acuerdas dónde fue?

N. Aqui ( $4 ; 4$ años).

El uso de las preposiciones, además de ser escaso («a», «con» y «en») suele ser inadecuado: bien no aparecen cuando su uso es necesario, o bien las usa incorrectamente, como se observa en los siguientes ejemplos:

- yo tengo un tren a casa ( $4 ; 4$ años).

- yo soy más grande con mis amigos (4;4 años).

Las conjunciones aparecen como una forma peculiar de la expresión verbal del niño y no como nexos entre palabras u oraciones, por ejemplo:

- que se cayó ( $4 ; 4$ años).

- ¿y el toro? (4;4 años).

\section{Análisis de las inadecuaciones estructurales del lenguaje}

Sintácticamente, el niño presenta errores en la construcción de sus enunciados y los manifiesta de las siguientes formas:

a Concordancia de número:

Como se puede observar en el siguiente ejemplo, el niño hasta casi los cuatro años no es capaz de concordar correctamente el número:

A. ¿Qué vas a hacer?

N. Un gatitos (3;11 años).

b. Omisión de elementos morfológicos:

Como es el caso de la omisión verbal, que puede observarse en el ejemplo siguiente:

A. ¿Sabes quién te llevó al cine?

N. Sí.

A. Dímelo.

N. Solo/solo (fui solo) (3;11 años).

O bien de la omisión de pronombre que aparece en los siguientes mensajes-tipo:

N. Mamá/() ha tirado este guau (el perro ha tirado un libro) $(3 ; 11$ años). 
A. ¿Lo ha tirado?

A. ¿Qué está haciendo?/aquí hay una niña/¿qué está haciendo?

N. Levantando () (4;4 años).

A. Levantándose.

c. Adición de elementos de categorias morfológicas:

Este tipo de errores aparece en algunas categorías, como pronombre, preposición y advervio:

Pronombre:

A. ¿Esto qué es?

N. Una me casa ( $4 ; 4$ años).

Preposición y Adverbio:

A. A ver si llegas/se sujeta muy mal éste.

N. Pues/tengo que sujetar a dónde abajo ( $4 ; 4$ años).

Todos estos errores muestan que el niño posee una noción mínima sobre la estructura sintáctica, su expresión presenta formas lingüísticas estereotipadas y automáticas, aprendidas en determinados contextos. El niño muestra poca capacidad generativa y de generalización de estas expresiones para adaptarlas a otros contextos nuevos.

\section{Análisis de la evolución lexical}

Comparando la evolución lexical, encontramos que en el caso de Carlos (el niño que urilizamos para la comparación) pasa de una producción de 170 palabras distintas a los dos años a más de 1.200 a los tres años -a partir de los cuales ya es imposible contolar su caudal lexical-, mientras que Miguel no ha alcanzado las 200 a los cuatro años y cuatro meses, por lo cual podemos concluir que este niño presenta un ritmo lento en la adquisición y desarrollo lexical.

\section{Análisis pragmático del lenguaje}

En este aspecto del lenguaje la diferencia más evidente con respecto a otros niños radica en el momento de aparición de las distintas categorías pragmáticas, más tardío en el caso de Miguel, ya que no aparecen en su totalidad hasta los cuatro años. Además, como se observa en la Tabla II el niño utiliza en un primer momento una cantidad superior de mensajes de tipo fático, frente a mayor uso de la función enunciativa en otros niños; aunque aparece un progresivo aumento de esta función ( $4 ; 4$ años), disminuyendo de forma paulatina la fática. En este sentido, la expresión lingüistica de Miguel se va aproximando a la forma del lenguaje de los otros niños, cuyo modo de uso más habitual es, como ya se ha señalado, precisamente la enunciativa (Shum, 1988) (Gráfico 2). Su pobre capacidad sintáctica y lexical, como se desprende del análisis de su producción lingüístic a, unido a la necesidad comunicativa que presenta en este momento, generada a través de la intervención realizada con el niño para habilitar el lenguaje, explicaría la alta frecuencia de la función fática y nos indica que en un primer momento Miguel utiliza el lenguaje para conseguir contacto social.

Dentro de la categoría enunciativa no aparecen mensajes de referencia a objeto ausente (ROA), diferencia evidente con respecto a otros niños que ya la usan de 


\section{5}

TABLA II

Evolución de las funciones pragmáticas del niño (puntuación porcentual)

\begin{tabular}{|c|c|c|c|c|c|c|c|c|c|c|}
\hline \multirow{3}{*}{ EDAD } & \multicolumn{5}{|c|}{ ENUNCIATIVA } & \multicolumn{4}{|c|}{ DEMANDA } & \multirow{3}{*}{$\begin{array}{c}\mathrm{FA}- \\
\mathrm{TI} \\
\mathrm{CA}\end{array}$} \\
\hline & \multicolumn{2}{|c|}{ DENOM OBIETOS } & \multicolumn{3}{|c|}{ REFER. ACCIION } & \multirow{2}{*}{$\begin{array}{c}A C C I O N \\
Y O B J .\end{array}$} & \multicolumn{2}{|c|}{ INFORMAC. } & \multirow{2}{*}{$\begin{array}{c}\text { AYU- } \\
\text { DA }\end{array}$} & \\
\hline & PRES. & ALSEN. & PRES. & PAS. & FUTURO & & INT AB. & INT.CER. & & \\
\hline $3 ; 2$ & 21,69 & & 2,11 & & & 2,64 & & 1,05 & & 36,50 \\
\hline $3 ; 11$ & 28,11 & & 4,20 & 1,48 & 0,99 & 2,47 & 4,70 & 3,96 & & 37,87 \\
\hline $4 ; 4$ & 52,60 & & 8,09 & 0,57 & & & & 8,09 & & 24,27 \\
\hline
\end{tabular}

Figura 2

Distribibución de las funciones pragmáticas en la producción lingüistica del niño

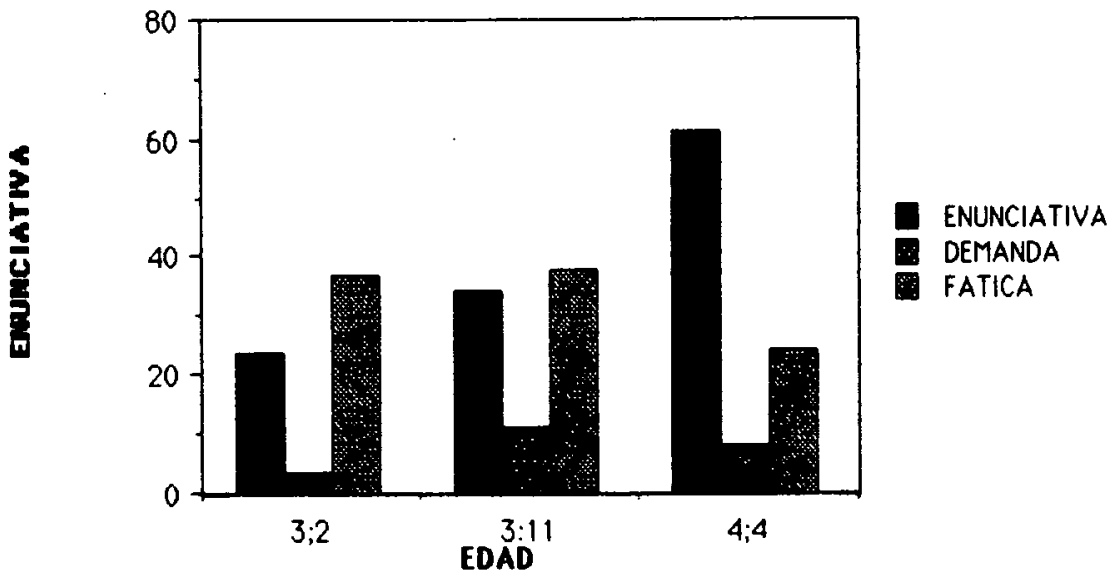

forma regular a los dos años de edad, manifestando, de este modo, un retraso notable en el lenguaje, así como en el desarrollo de la capacidad de representación, ya que no muestra conocer el concepto de ausente-presente. Este hecho, unido al desconocimiento de ciertas propiedades y funciones de los objetos que se manifiestan a través de la expresión lingüística, nos muestra un bajo nivel de desarrollo cognitivo del niño. Esto se evidencia en los siguientes ejemplos:

a. Referido a características del mundo físico:

A. ¿De qué color es ésta? (el adulto muestra al niño un objeto verde).

N. Amarillo (4;4 años).

b. Referido al conocimiento del cuerpo:

A. ¿Esto qué es? (señalando el cuello de un muñeco dibujado).

N. Cuerpo.

A. No/este trozo/este trocito, ¿qué es?

N. Tripa ( $4 ; 4$ años).

c. Referido al conocimiento espacial:

A. Cuéntame, ¿está debajo de las flores o encima? (el objeto señalado se encuentra encima).

N. Abajo ( $4 ; 4$ años). 
En cuanto a la categoría de demanda, puede decirse que hay un aumento en el uso de la demanda de información, sobre todo en forma de interrogación cerrada, y que no aparece la demanda de ayuda. Comparando con niños cuyo desarrollo ès adecuado, encontramos que éstos utilizan más la demanda de objeto y acción (DOA), Shum y otros, 1988a. Esta diferencia podría deberse a una estrategia comunicativa y de aprendizaje específica de este niño.

Se observa, además, una alta frecuencia y porcentaje de inadecuación pragmática, propia de niños que presentan un bajo nivel de desarrollo linguiístico (Shum, 1988), que hace sospechar que existen problemas en la comprensión del lenguaje del adulto por parte del niño, o de comprensión de las características del contexto, pero en cualquiera de los casos, manifiestan un desarrollo lingüístico deficitario.

\section{DISCUSION Y CONCLUSIONES}

Como se observa en el análisis anterior, el lenguaje de Miguel, tanto a nivel sintáctico, semántico como pragmático, manifiesta diferencias evidentes con respecto a niños con un desarrollo lingüístico adecuado. Además de presentar una menor frecuencia en el uso de las categorías morfosintácticas y pragmáticas, existe un retraso en el momento de adquisición de las mismas. El niño, como se ha indicado con anterioridad, no presentaba lenguaje verbal hasta en torno a los tres años de edad,y esto, fruto de una intervención de habilitación del lenguaje, lo cual indica un notable retraso en su desarrollo.

Durante el período de estudio, se observa que el niño presenta una mejora progresiva, sobre todo en el aspecto funcional del lenguaje. Como se indica en el análisis pragmático, esta mejora se manifiesta con el aumento del número de mensajes iniciados por el niño y el aumento de la función fática. Dada la característica de esta función -meramente interactiva- nos hace suponer que aparece una evolución de la competencia comunicativa (al menos en lo que se refiere a la necesidad del niño de comunicarse con su interlocutor), mientras que presenta todavía un déficit del desarrollo lingüístico, que le impide la producción de aquellas funciones de uso que exigen mayor intervención estructural y mayor riqueza semántica. Paulatinamente, y ya después de la adopción ( $4 ; 4$ años), se normaliza el uso del resto de las funciones pragmáticas, aunque no alcanza el nivel (frecuencia y variedad) de niños con desarrollo favorable, ejemplificado por el caso de Carlos. Esto evidencia que el nivel de desarrollo de Miguel todavía dista del de otros niños de su edad cronológica (su desarrollo es equivalente a niños de dos años), ya que el niño es incapaz de transformar los enunciados en función de la exigencia del contexto comunicativo, y de generalizar sus conocimientos lingüísticos a otros contextos interactivos. No ocurre, sin embargo, lo mismo con respecto a los trastornos orgánicos (peso, talla, raquitismo, etc.) que se detectaron en el comienzo del estudio. Esto significa que las secuelas físicas de los malos tratos tienen un mejor pronóstico de recuperación -siempre que el niño no haya superado el período crítico de dicho desarrollo- que las de tipo psicológico y, principalmente, el lenguaje.

Desde el momento del nacimiento, el niño se encuentra inmerso en un contexto social, dentro del cual se desarrolla. Pero dicho contexto en sí mismo no constituye la condición suficiente para una favorable evolución infantil, sino que es necesario que reúna una serie de características que se materializan en la adecuada interacción diádica adulto-niño. Desde el primer momento, aparece, en el caso que nos ocupa, ya un inconveniente importante. Miguel hasta pasados los dos años de edad no sólo no 
tenía una adecuada relación interactiva-afectiva, sino que, por el contrario, recibía malos tratos físicos y psíquicos. Posteriormente, su vida institucional, aunque mejora en los aspectos materiales, presenta aún deficiencias de tipo relacional, en el sentido, como ya es sabido, de no favorecer el desarrollo afectivo.y social infantil (Loutre, 1972; López Sánchez, 1981; Shum, 1986, etc.). La propia estructura de la institución no le proporciona la interacción comunicativa de forma adecuada que el niño necesita. Es decir, el adulto no cumple, en el proceso interactivo adulto-niño, la función de organizador de las experiencias infantiles y, a su vez, instruye y proporciona nuevas experiencias a éste para facilitar el adecuado desarrollo, en el sentido que plantea Bruner (1986). Por otra parte, el niño al no recibir la estimulación ni afectiva ni cognitiva necesaria, no puede incorporarse a la vida social, ya que carece de la actividad autónoma que lo posibilita. En este período Miguel presenta un conjunto de trastornos psicomotores, del lenguaje y sociales, que evidencian la incidencia negativa de estos factores en su desarrollo.

Después de la adopción, Miguel encuentra un entorno vital que puede considerarse adecuado. Sin embargo, aunque aparece una mejora notable en todos los niveles (socio-afectivo, comunicativo, etc.) mantiene un importante déficit, lingüístico y cognitivo, como ya se ha señalado con anterioridad.

A través de este estudio empírico, podemos llevar a cabo ciertas reflexiones sobre la necesidad de la adecuación de las pautas de interacción diádica temprana en el desarrollo infantil. Cuando no se dan de una manera correcta aparece un conjunto de déficits de desarrollo (tanto físico como psicológico) que incide de forma negativa en la evolución de las funciones psicológicas superiores (cognitiva, lingüística, etc.), y a la larga supondrán un inconveniente en el desarrollo infantil, que en muchos casos no podrá ser superado convenientemente. Esto nos lleva a plantear la necesidad de una intervención precoz cuando se detectan casos de malos tratos y abandono, asimismo, una revisión del funcionamiento de las instituciones de protección de menores, sobre todo en el aspecto comunicacional adulto-niño.

\section{Referencias}

AINSWORTH, M. D. S. (1969). Object relations, dependency and attachment: a theorical review of infant-mother relationship, Child Development.

BowBly, J. (1969). Attachment, Londres, Hogarth.

BOWBLY, J. (1976). El vínculo a fectivo, Buenos Aires, Paidós.

BruNer, J. (1984). Acción, pensamiento y lenguaje, Madrid, Alianza Psicología.

BRUNER, J. (1986). El habla del niño, Barcelona Paidós.

CAMPBELL, L., Y W WLES, R. (1976). El estudio de la adquisición del lenguaje, en Lyons, J. (ed.). Nuevos horizontes de la lingiǘstica, Madrid, Alianza Universidad.

DORE, J. (1978). Requestive sisytems in nursey school conversations: analysis of talk in its social context, en Campbell, R., y Smith, P. (ed.). Recents advances in the psychology of language: Language development and mothers-child interactions, Nueva York, Plenum Press.

FRENCH, P., Y MALURE, M. (1981). Teacher's questions, pupil's answers: an investigation in the infant classroom, en Stubbs, M., y Hillier, H.: Readings on language, schools and classrooms, Londres y Nurva York. Methuen.

GARDNER, L. I. (1976). El enanismo por privación, Psicobiología Evolutiva, Scientific America, Fontanella, 121-128.

IVANOV-SMOLENSKY, A. G. (1951). Acerca del estudio de la actividad conjunta del primer y segundo de señales. Citado en Luria, A. R., y Yudovich, F. I. A., Lenguaje y desarrollo intelectual en el niño, Madrid, Pablo de Río.

KAYE, K. (1986) La vida mental y social del bebé, Barcelona, Paidós.

KRANOSGORSKY, N. I. (1954). Estudio sobre la actividad nerviosa suterior en los animales y en el bombre. Citado en Luria, A. R., y Yudovich, F. I. A., Madrid.

LOPEZ SANCHEZ, F:(1981a). Los orígenes de la sacialización: la vinculación afectiva, Infancia y Aprendizaje, 15, 53-66. 


\section{8}

LOPEZ SANCHEZ, F. (1981b). Niños en casa-cuna, Infancia y Aprendizaje, 16, 81-94.

LOUTRE, N. (1981). El retraso de origen afectivo: nueva orientación en el enfoque de las deficiencias intelectuales y revisión crítica de las literaturas sobre la carencia de cuidados maternos, Infancia y Aprendizaje, 54-65.

LoUTRE, N., (1981b). Adaptación recíproca niño-nodriza, Infancia y Aprendizaje, 16, 95-100.

LuRIA, A. R., Y YUDOVICH, F. I. A. (1978). Lenguaje y desarrollo intelectual en el niño, Madrid, Pablo del Río.

MARDOMINGo, M. J. (1981). Efecto de la institucionalización sobre la conducta del niño durante los tres primeros años de vida. V Simposio Español de Pediatría Social, Tenerife.

MilNeR, J. S., Y Wimberley, R. C. (1979). An Inventory for the identification of child abusers. Journal of Clinical Psychology, 35 (1), 865-866.

MOERK, E. L. (1985). Procedures and processes of first language teaching and learning, actas de First International Congress of applied Psycholinguistics, Barcelona.

Newson, J. (1977). La interacción madre-bijo: una descripción sistemática e intersujetiva, en Perinat, A. (comp.), 1986: La comunicación preverbal Barcelona, Ediciones Avesta.

POWELl, D. R. (1980). Personal social network as a focus for primary prevention of child mistreatment. Infant Mental Health Journal, 1 (4), 232-239.

PAUl OChOROTORENA, J., y otros (1988). Maltrato y abandono infantil: identificación de factores de riesgo, Servicio Central de Publicación del Gobierno Vasco.

ROBERTSON, K. R., Y MILNER, J. S.(1987). An inconsistency Scale for the child abuse potential inventory, Psycbological Report, 60, 699-703.

ROSENGARDT, G. L. (1947). El babla y el desarrollo de la percepción infantil, citado en Luria, A. R., y Yudovich, F. I. A.

SNOw, K. (1977). Mothers' speech research: from input to interaction, en Sow y Ferguson (eds.): Talking to children, Londres, Cambridge University Press.

SNow, K,. Y Ferguson (1977). Talking to children, Londres, Cambridge University Press.

SHUM, G. (1986). Psicolingüistica aplicada al estudio de la adquisición del lenguaje en niños institucionalizados $y$ no institucionalizados. Tesis doctoral, Madrid, Universidad Complutense.

SHUM, G. (1988). La influencia del contexto de la interacción en la adquisición del lenguaje, Infancia y Aprendizaje, 43, 37-53

SHUM, G., y otros. (1988a). Lenguaje y adquisición de conocimiento: un estudio comparativo, Estudios de Psicología, 33-34, 69-81.

SHUM, G., y otros. (1988b). ¿Cómo se adquieren y usan los términos deícticos en lenguaje español. Un estudio longitudinal. Infancia y Aprendizaje, 48, 45-64.

SCHAFFER, H. R. (1977). Early interactive development en Motber infant interaction. Londres, Academic Press.

SCHAFFer, H. R., CROOK, CH. K. (1981). El papel de la madre en el desarrollo social. Infancia y Aprendizaje, 15, 19-37.

TIZARD, P. (1972).Enviromental effects on language development: a study of young children in longstay residental, Cbild Development, 43.

TURNER, J. (1980). El niño ante la vida: enfrentamiento, competencia y cognición. Madrid, Morata.

VIGOTSKI, L. S. (1934/1973). Pensamiento y lenguaje, Buenos Aires, Pléyade.

VIGOTSKI, L. S. (1986). Aprendizaje y desarrollo intelectual en la edad escolar, en VV. AA. Psicologia y Pedagogía, Madrid, Akal.

VILA, I. (1985). La competencia comunicativa en los 2 primeros años de vida. Tesis doctoral, Barcelona, Universidad Central.

WaLLON, H. (1970). La evolución psicológica del niño, Buenos Aires, Nueva Visión.

WELLS, G. (1981). Language as interaction, en VV. A A. Learning through interaction. Londres, Cambridge University Press, 1-115.

\section{Extended Summary}

For adequate child development a favourable physical backgroud is necessary together with a good interactive context. In early infancy, the adult not only takes care of the child, but also guides and organizes him/her; helping him/her to learn and acquire different knowledge and skills. In early adult-infant interaction the adult also provides the emotional support the child needs which is essential for fostering a healthy development.

Early infant communication patterns are closely related to the family environ- 
ment. If this deteriorates (e.g, such as in cases of child abuse and neglect) adult-child caretaking and interactive patterns become inadequate. The child's growth and development suffers, particularly her/his language acquisition.

The present research is a longitudinal study on the language acquisition and development of a young child abused from birth. The study covers two different periods: from the age of 2;6 yrs., when he was living in an infant protection institution, to the age of 4;6 yrs., 6 months after he was adopted.

The aim of the study was to collect data on language acquisition patterns. For this purpose, we observed the acquisition of verbal levels and models in our subject. Also the child's language development was compared with that of normally developing child. Finally, special attention was paid to those linguistic elements which posed the greatest difficulty to the subject in terms of both language production and comprehension.

The data was recorded using audio-visual equipment. Language production was analyzed from three levels: Syntactic, semantic, and pragmatic.

Results show that after six months living with his adoptive parents, the child was able to overcome most of this physical problems. It seems that when his family environment changed his organic deficits were normalized. This change is also explained by more rewarding adult-infant interactions. Unfortunately, this is not the case with his language development which was still very poor in all three levels: Syntactic, semantic, and pragmatic.

In summary, the syntactical structure of messages expressed by the child were very simple and inflexible. His morphological acquisition was limited, and the contents of his utterances poor. Moreover, through the pragmatic analysis it was observed that the infant did not know how to correctly transform his utterances to different communication contexts. The child's linguistic level, both receptive and expressive, was also very limited. 